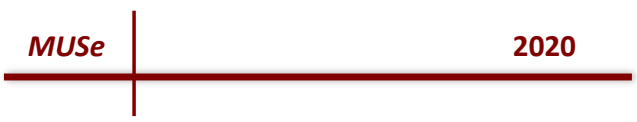

\title{
Is Young Blood the Cure to Aging?
}

\section{Kennedy Lacombe}

\begin{abstract}
Recent studies on parabiosis between young and old animals have discovered that young blood has a powerful rejuvenating effect on skeletal and cardiac muscles, as well as on the brain of older animals. These results suggest that there is something in the young systemic environment that may have anti-aging properties. The results have recently been attributed to a circulating protein by the name of GDF11. These results have garnered a lot of attention with the hopes that this protein could be the "cure" for aging. With the increasing attention that this concept has gathered, it is no surprise that GDF11 also raises a lot of ethical concern and controversy. More studies have come out in opposition, suggesting that GDF11 may have been wrongly attributed to the rejuvenating results, and may actually be doing more harm than good. Recent controversies, related challenges, and a future outlook are highlighted and examined further.
\end{abstract}

Reversing the signs and effects of aging has been sought after by people, including scientists, for as long as can be remembered. Cosmetic surgery is slowly becoming one of the largest industries in the world, because people are concerned with looking eternally youthful (Plastic Surgery Statistics, n.d.). Not only can these results greatly contribute to the superficial needs of the general public, but understanding the mechanisms of how we age can also help prevent age-related diseases (Ma et al., 2019). It is no surprise that as we age, our bodies slowly start to deteriorate. Our bones become frail, and the skeletal muscles surrounding them become weak and lose their flexibility (Egerman et al., 2015). Cardiac muscles also degenerate slightly, and heart valves become thick and stiff, resulting in our hearts having to work harder to pump blood (Egerman et al., 2015). Additionally, our brains undergo a number of changes, causing numerous impairments in cognitive functioning (Egerman et al., 2015). The mechanism for these degenerative effects, however, is unknown (Egerman et al., 2015).

The desire to discover the mechanism, as well as a longing to find a 'cure' for aging, has led to numerous studies looking for the factor(s) responsible for aging (Egerman et al., 2015). Researchers have begun to believe that a circulating protein, growth differentiation factor 11 (GDF11), which is known to regulate diverse cellular processes, is the cause of these agerelated issues (Egerman et al., 2015). Finding a circulating factor responsible inspires hope for novel approaches to treating age-related diseases in humans, and as a result, some scientists have attempted to use this protein to slow or even reverse the effects of aging (Ma et al., 2019; Egerman et al., 2015). However, increasing reports have shown contradictory data, questioning the capacity of GDF11 to reverse age-related degeneration (Ma et al., 2019; Egerman et al., 2015).

Transfusion of blood, or of its components, is not a new concept, and has been used as a rejuvenation method since ancient times (Conese et al., 2017). For example, physicians used 


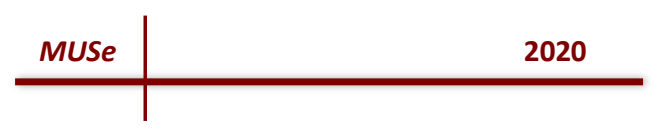

to prescribe bathing in or drinking young blood during the $17^{\text {th }}$ and $18^{\text {th }}$ centuries for rejuvenation purposes (Conese et al., 2017). Even stories about Dracula have him drinking young blood in order to live forever. Conboy et al., (2003) decided to put this idea to the test and see if there was any legitimacy to this old, gruesome technique. Parabiosis is described as a shared circulatory system, and GDF11 became the target of multiple studies after Conboy et al. (2003) performed a parabiosis study on mice. In this case, two mice, one young and one old, were sown together so that blood would circulate through both of them (Conboy et al., 2003). This exposed the old mice to all factors present in the young blood, and vice versa (Conboy et al., 2003). Conboy et al. (2003) performed this study to determine if there were any systemic factors in young blood not present in old mice that could be the cause of the effects of aging. Using immunofluorescence to better examine the condition of the muscle cells of both mice, Conboy et al. (2003) were able to examine the skeletal muscle conditions of mice both before and after the procedure.

The results were shocking. They showed that the older mice began to regenerate tissue stem cells at a higher rate than before, while the young mice began to degenerate at a faster rate (Conboy et al., 2003). These results suggest that there are systemic factors in the blood of young animals that can modulate the signaling pathways critical to the regeneration of tissue cells (Conboy et al., 2003). In addition, the results imply that the negative effects of aging are reversible through the alteration of these systemic factors (Conboy et al., 2003).

Based on the results from the Conboy et al. (2003) study, Sinha et al. (2014) looked more specifically into what the regenerative factor for skeletal muscles in young blood could be. After performing another parabiosis experiment that garnered the same results as the Conboy et al. (2003) study, Sinha et al. (2014) performed a gel electrophoresis assay looking at a number of different possible proteins in both old and young mice. They discovered that among the proteins found, GDF11 levels decreased significantly in older mice (Sinha et al., 2014).

Sinha et al. (2014) followed up their study by injuring old mice, treating half of them with GDF11, and using the other half as a control. They found that supplementation of GDF11 in aged mice restored and regenerated muscle cells and increased the mean size of muscle cells of young control mice to $92 \%$ (Sinha et al., 2014). However, it did not alter the muscle fibers of uninjured young or old mice (Sinha et al., 2014).

Seeing the emerging evidence that GDF11 might influence aging skeletal muscles encouraged further research into the protein, including its effects on other parts of the body. More specifically, Loffredo et al. (2013) looked into how, and if, GDF11 could reverse the signs of an aging heart. Loffredo et al. (2013) studied the influence of circulating factors using another parabiosis study, and after four weeks looked at the results. They found that after one month of exposure to the circulation of young mice, cardiac hypertrophy, the thickening and stiffening of the heart muscle, in old mice dramatically reverted (Loffredo et al., 2013).

As with skeletal and cardiac muscles, the nervous system also experiences age related degeneration of neural stem cells, resulting in reduced blood flow and neurogenesis, which is defined as growth of the nervous tissue (Katsimpardi et al., 2014). Previous research on GDF11 found that it is essential for embryonic development and neurogenesis, and also participates in multiple biological processes of the CNS (Ma et al., 2019). Some researchers have argued that restoring the functionality of the specific pathway responsible for the regeneration of cells using GDF11 should counteract some of the negative effects of aging on the brain (Katsimpardi et al., 


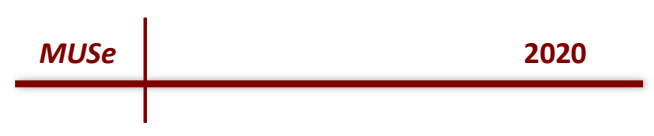

2014). Katsimpardi et al. (2014) tested this theory by performing a study similar to Sinha et al.'s (2014) in which they treated older mice with GDF11. The study differed only in that instead of looking at skeletal muscles like Sinha et al., they looked at brain capillary endothelial cells. Once again, Katsimpardi et al. started their study with another parabiosis examination to ensure that the circulation of young blood actually did help regenerate the older mouse, and the results once again confirmed that it did (Katsimpardi et al., 2014). Katsimpardi et al. (2014) further noted that GDF11 was able to increase blood flow and neurogenesis in aged mice. They did find, however, that the results were not as strong and effective as with parabiosis itself, but were significant regardless (Katsimpardi et al., 2014).

Even given all these promising studies and results, there were many controversies surrounding GDF11 and its role in combating the effects of aging. In fact, several studies looking specifically at GDF11 before 2003, concluded that it does not decrease in concentration with age, and that it may contribute to muscle weakness (Rodgers \& Eldridge, 2015). In addition, it was found that Loffredo et al. (2013) and Sinha et al.'s (2014) studies were not reproducible, and they may have been attributing their results to the wrong factor, due to a faulty recording procedure (Rodgers \& Eldridge, 2015). Many researchers attempted to recreate the studies that found GDF11 levels decreased as we age, but were unable to do so, either because of the methods used, or the ways in which GDF11 was measured (Hinken et al., 2016).

Given that there have been two drastically different and contradictory research results for the role and expression of GDF11 in the body as we age, a number of scientists questioned the methods used, and attempted to reproduce studies. For example, Egerman et al. (2015) demonstrated that the methods used, more specifically the western blot analysis used in Loffredo et al. (2013) and Sinha et al.'s (2014) studies, were actually not specific to GDF11. Consequently, GDF11 has been surrounded by controversy, attributed in part to challenges associated with the accurate measurement of its circulating levels and their dissociation from circulating myostatin levels (Schafer et al., 2016). In both Loffredo et al. (2013) and Sinha et al.'s (2014) study, the antibody used to bind to GDF11 (in order to determine its expression in the body) also binds to the protein myostatin, and one cannot distinguish between the two (Egerman et al., 2015). Myostatin is known to diminish as we age, and so not being able to differentiate between myostatin and GDF11 could be the reason for the results suggesting that GDF11 decreases as well (Egerman et al., 2015).

Egerman et al. (2015) found a way to specifically detect levels of GDF11 in the blood of humans using an immunoassay technique, and tested it on humans, rather than mice. When comparing the expression of GDF11 in 60-year-olds versus 20-30-year-olds, they found that there were significantly higher levels of GDF11 in older humans than in younger humans (Egerman et al., 2015). This coincided with the earlier studies looking at GDF11 (Egerman et al., 2015). To further solidify their findings, they reproduced the same study Sinha et al. (2014) did, in which they administered GDF11 treatment or a control treatment (in which there should be no effect) to aged mice for 7 days (Egerman et al., 2015). The only difference was that they used their immunoassay technique to measure the expression of GDF11 (Egerman et al., 2015). This experiment concluded that GDF11 levels in older animals were not diminishing, and there were no differences when observing regenerative capacity of skeletal muscles between young and old mice (Egerman et al., 2015). 


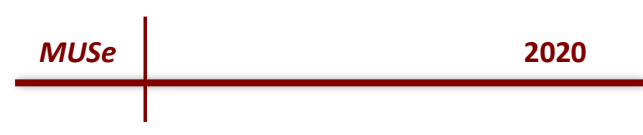

Schafer et al. (2016) added more support to the result that GDF11 levels actually increase as we age by using their own specific immunoassay technique, and once again testing on humans. Schafer et al. (2016) demonstrated that people with higher levels of GDF11 were more likely to be frail and have diabetes or prior cardiac conditions. Additionally, those with high levels of GDF11 experienced higher disease burden, reduced resiliency, and elevated postoperative risk (Schafer et al., 2016). In humans, it was also demonstrated that levels of GDF11 do not significantly differ between men and women (Schafer et al., 2016). Schafer et al.'s (2016) study further revealed that myostatin levels decreased as people aged, and were the lowest in those experiencing sarcopenia, or age-related muscle degeneration.

Egerman et al. (2015) finalized their research by performing a study in which they increased GDF11 levels in young mice (which in their study, had lower levels of GDF11 than older mice) and showed that GDF11 was harmful toward muscle repair in young mice (Egerman et al., 2015; Rodgers \& Eldridge, 2015). They noticed that muscle fibers in GDF11-treated mice actually began to shrink due to a decreased number of muscle cells, and in regenerating muscle, GDF11 treatment was associated with a greater frequency of small fibers (Egerman et al., 2015). Given these studies, it is possible that GDF11 could even be the cause of some of the effects of aging, and therefore we should possibly be looking at ways to block it, rather than increasing it (Egerman et al., 2015). Clearly, further investigation is needed to fully clarify whether or not, and under what conditions, GDF11 is a factor that beneficially regulates muscle activity during aging.

Not only were some methods in earlier studies faulty, but information from these studies was also taken out of context. For example, Conboy et al. (2003) from the first parabiosis study did an interview in which they addressed some of their concerns with the way their study had influenced other researchers (Chen, 2019). While there was significant evidence suggesting that there could be a factor in young blood that could potentially reverse the signs of aging, Conboy et al. (2003) urged the public to take the results with a grain of salt (Chen, 2019). They explained that during a parabiosis study, not only do you share the same circulatory system, but you also share two of every organ (Chen, 2019). The old mouse in their study could have seemed restored and youthful because it was also able to utilize a young heart, young lungs, etc. (Chen, 2019). In addition, a very important, but usually neglected, result from their study was that the young mice began to deteriorate and die off when sown to older mice (Chen, 2019).

Conboy et al. (2003) also informed the public that even though their study did show positive results, and that there is reason for further research into what systemic factors in young blood could be causing the regeneration of old tissue, they warned that there are also many possible side effects of blood transfusions if not administered carefully (Chen, 2019). While the mice that were part of the study were inbred and very compatible, people are not genetically matched (Chen, 2019). Careless use of blood transfusion could therefore result in dangerous side effects, such as allergic reactions, infection, blood transmitted diseases, etc. (Chen, 2019). There are many issues that can arise with blood transfusions in humans if the proper precautions aren't taken to assure the compatibility of the blood from the donor and the recipient (Pardi, 2017). Conboy et al. (2003) emphasized that they were purely in the research phase, stating that there are a lot of variables to consider when administering blood transfusions, and ignoring or neglecting these can lead to serious consequences (Chen, 2019). 


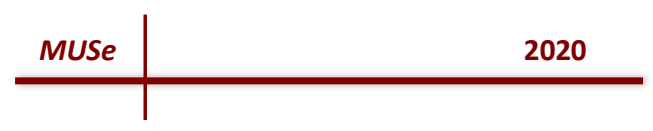

For humans, eternal youth has been sought after for centuries. With the societal standards of beauty changing, and with a growing proportion of the population aging, limiting the effects of aging is more important now than ever before. That being said, small advancements towards this create a lot of publicity and, if in the wrong hands, can be detrimental. The media got ahold of these studies and, when transmitting the information to the public, only used the eye-catching and interesting results (Pardi, 2017). The media blew up with the story that scientists had found the "cure" for aging in young blood, while neglecting to mention the procedures and uncertainties behind the research, or that it had not been tested on humans (Pardi, 2017).

Neglecting to give the whole truth of these studies was misleading and could have caused people to engage in risky activity when there is still research (including human trials) to be done. The exaggerated claims made in the media can be dangerous on a number of levels. One example is a large company by the name of Ambrosia (Ivy Plasma, n.d.).

Ambrosia began selling human blood plasma from younger donors with the promise of anti-aging and rejuvenating effects to people above the age of 35 for $\$ 8,000$ (Ivy Plasma, n.d.). Not only was it extremely unethical to ask people to pay large sums of money to unknowingly be part of the first human trials, but the company also made false promises to their customers (Ivy Plasma, n.d.) The founder, Jesse Karmazin, who is not a licensed doctor, made positive claims about the results of these human trials, without posting any of the scientific results as proof (Ivy Plasma, n.d.). Karmazin also held back information about the specifics of the tests themselves, and it is still unclear how they were done and how they affected the patients (Ivy Plasma, n.d.). Companies such as Ambrosia exploit innocent, gullible people, and are the reason the FDA had to send out a report stating that this procedure was not FDA approved because of lack of research and unknown long term results (Gottlieb, 2019).

Research studies utilizing parabiosis have brought humans closer to the 'fountain of youth' than ever before, but there are still a number of steps to take, and a number of studies to be done, before treatments are available (Hofmann, 2018). Future research should consider the idea that the rejuvenating factor (or a combination of factors) may not be limited to the blood (Ma et al., 2019). Other factors should be considered because substances found in plasma come from both blood cells and peripheral organs (Ma et al. 2019). As is well understood, blood is composed of blood cells and plasma (Ma et al. 2019). It would be appropriate to research the effects when using only blood cells, or only plasma, or a combination of both (Ma et al. 2019).

Also, using research done on mice to men requires careful consideration (Hofmann, 2018). Parabiosis and blood transfusions pose a number of problems, and the concept of using internal substances in some people as a means to rejuvenate others calls for ethical reflection (Hofmann, 2018). Considering that blood transfusion is a clinically approved technique, transfusion of young blood could potentially be a novel way to combat or prevent aging (Ma et al., 2019). However, it is worth mentioning that if this functions properly, there is going to be a huge demand for young blood, for both recreational and medical use (Ma et al., 2019). This would create a demand that will likely not be able to be kept up with, which could possibly result in other dangerous outcomes (Ma et al., 2019). Promising parabiosis research indicates that we may be closer to eternal youth than ever before (Hofmann, 2018). But, it is far too early to assess the implications for clinical practice, health policy, and society (Hofmann, 2018). 


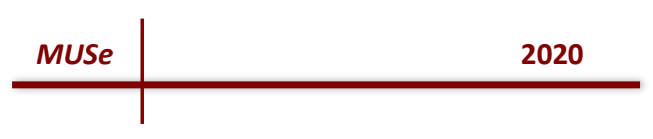

\section{References}

Chen, A. (2019, March 26). Retrieved October 2, 2019, from https://www.theverge.com/2019/3/26/18280967/young-blood-food-drug-administrationhealth-aging-longevity-parabiosis-science.

Conboy, I.M., Conboy, M.J., Smythe, G.M., \& Rando, T.A. (2003). Notch-mediated restoration of regenerative potential to aged muscle. Science, 302(5650), 1575-1577.

Conese, M., Carbone, A., Beccia, E., \& Angiolillo, A. (2017). The fountain of youth: A tale of parabiosis, stem cells, and rejuvenation. Open Medicine, 12(1). https://doi:10.1515/med$\underline{2017-0053}$

Egerman, M.A, Cadena, S.M., Gilbert, J.A., Meyer, A., Nelson, H.N., Swalley, S.E., ... Glass, D.J. (2015). GDF11 increases with age and inhibits skeletal muscle regeneration. Cell Metabolism, 22(1), 164-174.

Gottlieb, S. (2019). Statement from FDA Commissioner Scott Gottlieb, M.D., and Director of FDA's Center for Biologics Evaluation and Research Peter Marks, M.D., Ph.D., cautioning consumers against receiving young donor plasma infusions that are promoted as unproven treatment for varying conditions. Retrieved from https://www.fda.gov/newsevents/press-announcements/statement-fda-commissioner-scott-gottlieb-md-anddirector-fdas-center-biologics-evaluation-and-0.

Hinken, A.C., Powers, J.M., Luo, G., Holt, J.A., Billin, A.N., \& Russell, A.J. (2016). Lack of evidence for GDF11 as a rejuvenator of aged skeletal muscle satellite cells. Aging Cell 15, 582-584. https://doi:10.1111/acel.12475

Hofmann, B. (2018). Young blood rejuvenates old bodies: a call for reflection when moving from mice to men. Transfusion Medicine and Hemotherapy, 45(1), 67-71. https://doi:10.1159/000481828

Ivy Plasma. (n.d.). Retrieved October 2, 2019, from https://www.ivyplasma.com/

Katsimpardi, L., Litterman, N.K., Schein, P.A., Miller, C.M., Loffredo, F.S., Wojtkiewicz, G.R., ... Rubin, L.L. (2014). Vascular and neurogenic rejuvenation of the aging mouse brain by young systemic factors. Science, 344(6184), 630-634. https://doi:10.1126/science.1251141

Loffredo, F.S., Steinhauser, M.L., Jay, S.M., Gannon, J., Pancoast, J.R., Yalamanchi, P., ... Lee, R.T. (2013). Growth differentiation factor 11 is a circulating factor that reverses agerelated cardiac hypertrophy. Cell 153, 828-839. https://doi:10.1016/..cell.2013.04.015

Ma, J., Gao, B., Zhang, K., Zhang, Q., Jia, G., Li, J., ... Cai, Z. (2019). Circulating factors in young blood as potential therapeutic agents for age-related neurodegenerative and neurovascular diseases. Brain Research Bulletin, 153, 15-23. http://doi:10.1016/j.brainresbull.2019.08.004

Pardi, D. (Interviewer), Conboy, I., \& Conboy, M. (Interviewees). (2017). Fight Aging. Retrieved from https://www.fightaging.org/archives/2017/02/an-interview-with-irina-and-michaelconboy-on-blood-factors-in-aging/

Plastic Surgery Statistics. (n.d.). Retrieved from https://www.plasticsurgery.org/news/plasticsurgery-statistics 


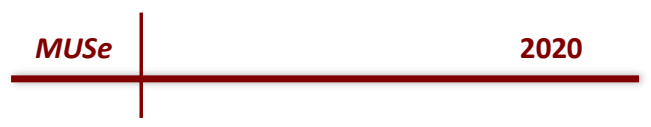

Rodgers, B.D. \& Eldridge, J.A. (2015). Reduced circulating GDF11 is unlikely responsible for age-dependent changes in mouse heart, muscle, and brain. Endocrinology, 156(11), 3885-3888. https://doi:10.1210/en.2015-1628

Schafer, M.J., Atkinson, E.J., Vanderboom, P.M., Kotajarvi, B., White, T.A., Moore, M.M. ... LeBrasseur, N.K. (2016). Quantification of GDF11 and myostatin in human aging and cardiovascular disease. Cell Metabolism, 23(6), 1207-1215. http://doi: $\underline{10.1016 / \mathrm{j} . \mathrm{cmet} .2016 .05 .023}$

Sinha, M., Jang, Y.C., Oh, J., Khong, D., Wu, E.Y., Manohar, R., ... Wagers, A.J. (2014). Restoring systemic GDF11 levels reverses age-related dysfunction in mouse skeletal muscle. Science, 344(6184), 649-652. https://doi:10.1126/science.1251152 